\title{
Silica Nanoparticles Induced Oxidative Stress in Different Brain Regions of Male Albino Rats
}

Manoj Kumar Maurya ${ }^{1}$, Raghev Langeh ${ }^{1}$, J Anuradha ${ }^{1}$, Rohin Sanjeevani ${ }^{2}$, R Sanjeevi ${ }^{1}$, Sandeep Tripathi ${ }^{1}$, Dushyant Singh Chauhan ${ }^{1 *}$

\author{
${ }^{1}$ Department of Advanced Science \& Technology, Faculty of Engineering \& Technology, Nims University Rajasthan, Jaipur- 303121, \\ India \\ ${ }^{2}$ Department of Microbiology, National Institute of Medical Sciences \& Research, Nims University Rajasthan, Jaipur-303121, India
}

DOI: $10.36347 /$ sajb.2021.v09i05.004 | | Received: 14.04.2021 | Accepted: 25.05.2021 | Published: 30.05 .2021

*Corresponding author: Dushyant Singh Chauhan

Abstract Original Research Article

Silica nanoparticles (SiNPs) are encapsulated in an ecological platform has been planned to supply many benefits, and blood vessels used as laser-tissue-soldering within the brain, and during the course of the degradation, SiNPs given to the discharged into an encircling of the brain tissue. The goal of this modern research was to examine likely to the silicon dioxide nanoparticles are categorized in the microglial cells and uptake the cellular mechanism(s), and additionally these impacts on autophagy and inflammatory cytokines, and also it's been found in depth applications in medical specialty and biotechnological fields, for example, bio imaging, medical diagnostics, gene therapy, drug delivery, photodynamic therapy biomolecule detection and bio-imaging. It has also been added to the raising of the industrial mechanism to the exposure of silicon dioxide nanoparticles, throughout the manufacturing, transportation, storage, and consumers that are obviously increased used by human exposure and environmental burden. The purpose of this work was to examine the neurotoxicity of two different dosages ( 40 and $80 \mathrm{mg} / \mathrm{kg}$ body weight) of silica nanoparticles (SiNPs) in male albino rats for 14 days by intraperitoneal exposure. The neurobehavioral and neurochemical changes were analyzed. Spontaneous motor activity, catalepsy, gait and muscle incoordination were assessed. There after the brain was removed for the examination of lipid peroxide level and antioxidant enzymes (SOD and CAT). Our results indicated the SNPs could passed from the blood-brain-barrier into the brain, and essentially it increases the concentration of lipid peroxide levels and reduce the activity of SOD and CAT and also its reduce the behavioral activity were seen. The maximum changes were observed in rats, who received $80 \mathrm{mg} / \mathrm{kg}$ body weight doses. It can be concluded that neuronal toxicity of silica nanoparticles could be related to the amount of the particles and the oxidative stress could be involved in cellular damage by lipid, protein, DNA and cognitive decline.

Keywords: Silica Nanoparticles, Brain, SOD, Oxidative stress.

Copyright (C) 2021 The Author(s): This is an open-access article distributed under the terms of the Creative Commons Attribution 4.0 International License (CC BY-NC 4.0) which permits unrestricted use, distribution, and reproduction in any medium for non-commercial use provided the original author and source are credited.

\section{INTRODUCTION}

Silica is the one name that given to a group of minerals that created from silicon and oxygen; there is the two most copious components within the earth's crust (Nakamura et al., 2015). Commonly silica dioxide is found within the crystalline state and infrequently did it realize in an amorphous state. It's made up of one atom of the silicon and two atoms of the oxygen than its statement is $\mathrm{SiO}_{2}$ (Sanchez and Sobolev, 2010).

Generally, it's most typically found in the nature as sand or quartz and it also found in the each part of the human body organ through the blood cardiovascular system to the transmission of nerve signals. Silica dioxide nanoparticles $\left(\mathrm{SiO}_{2}-\mathrm{NPs}\right)$ are unit getting used progressively in diagnosis, imaging, and drug-delivery for the central nervous system (Jong and Borm, 2008).

Nootropics area unit a large and structurally heterogeneous classes of drugs (also supplements, nutraceuticals, and purposeful foods) that improves at least one parts of mental functions, for example, working memory, motivation, and attention (Abbott, 2006). There's proof that silica dioxide nanoparticles revelation can also be connected to the enlargement of autoimmune diseases, for example, lupus, chronic renal diseases, scleroderma (systemic sclerosis), and rheumatoid arthritis.

The variety of pathologies related within the SiNPs revelation and it's unlikely that which have 
Manoj Kumar Maurya et al., Sch Acad J Biosci, May, 2021; 9(5): 139-144

common mechanism to blame for all the likely diseases (Cole and Sumnall, 2003). Several NPs have area unit which able for crossing or by passing the blood-brain-barrier (BBB) (Bourdonnec et al., 2013). Additionally, the mechanism of free radicals production and defended by the antioxidant will also be evaluate using the biochemical examinations. Many animal researches directly proved NPs might spread and collect within the brain parenchyma, together with the hippocampus and striatum. It was observed that, translocation of zinc oxide ( $\mathrm{ZnO})$ NPs into the following brain in vivo nasal administration in a Sprague Dawley male albino rat's perfect model (Ying-Mei et al., 2016). There are the mechanisms for the silica nanoparticles neurotoxicity; due to the size of silica nanoparticles are small that may simply reach within the brain and area unit concerned by the brain cells, for example, neurons and glia (Ungvari et al., 2011). The interaction between biological molecules and NPs, for example, DNA, Proteins and lipids, might has several results, containing oxidative stress; conformational alteration increases the membrane permeability mutation, ionic exchange disorder, signaling pathway activation, enzyme failure and new protein epitope exposure (Zhang et al., 2012). After interacting with nanoparticles, numerous intermediators discharged from the glia and neuroglia cell which includes the apoptosis, inflammation, and oxidative stress within the animal's brain, as a result of animal nervous tissue regeneration is proscribed, most of the damage nerve is irreversible (Kontoleontos et al., 2012).

The mechanism of nano neurotoxicity finds the interaction between the nanoparticles and the Bio systems is additionally determined through the category of nanoparticles. Soft nanoparticles, for example, liposomes and hard nanoparticles, for example, metal oxides irritate the bio system by the various mechanism and in reactive oxygen species (ROS), oxidative stress, are unit extremely reactive molecules that contain associate in an oxygen atom; Example: involve the superoxide radical $\left(\mathrm{O}_{2}\right)$ oxygen and $\left(\mathrm{H}_{2} \mathrm{O}_{2}\right)$ hydrogen peroxide (Yang and Li, 2014). Reactive Oxygen Species (ROS) areas are the unit which available in each cell, being made by the mitochondria, cytoplasmic oxidation process (Kim et al., 2015). The low ROS concentration is important to keep up the traditional physiological processes. For an excessive amount of manufacturing of Reactive oxygen species induced through the oxidative stress would be highly damaging (Pisani et al., 2015).

Silica nanoparticles inserted in an ecological stage has been proposed to suggest the various advantages, and when it once used in laser-tissue-soldering of blood vessels within the brain. Throughout degeneration, these nanoparticles area unit probably to be discharged in take encircling of the brain tissues (Zielinski et al., 2016). The effect of silica on solid tumor in vivo was restricting by its poor pharmacokinetics and dose-limited toxicity. A silica nanoparticle was direct to enhance the pharmacokinetics and enhance the antitumor effect of the arsenic trioxide and also the mesoporous silica nanoparticles loaded with the arsenic trioxide was joined with the polyacrylic acid (PAA-ATO-MSN) as a pH-responsive biomaterial on the active surface to achieve the discharge of drug in acidic microenvironment of tumor, rather than burst discharge the action in circulation (Xiao et al., 2016). Humans are exposed to nanoparticles from atmosphere and certain workplaces. Nanoparticles are characterized into two main types; combustion-derived NPs (e.g., diesel exhaust particles, particulate matters, and welding fumes) and made or designed the NPs.

In the modern study, our aim to assess the study of SNPs induced oxidative stress in different brain regions and also the effect of central nervous system (CNS) disorders on the human population is critical, it covering many of diseases with extremely disable behavioral and also social and cognitive deficits. The brain disorders functions area specifically dangerous as a result of they have an effect on showing emotionally, learning ability, and memory that unfolds and regresses as a particularly gets more older, Than that restricted capacity of self-repair mechanisms within the brain will leads to irreversible practicality, establishing disorders that don't have a serious impact on particularly lives, however still a location of several stress on health care resources (Gustavsson et al., 2011).

\section{MATERIAL AND METHODS Animals}

In the present study, a total number of male albino rats was 18 , its weight was $150 \pm 10$ grams which taken from NIMS University, Jaipur Rajasthan. The animals were separately housed in polypropylene cages in a room, which was maintained at a temperature of $22 \pm 20 \mathrm{C}$, relative humidity of $50 \pm 10 \%$ and $12 \mathrm{~h}$ light dark cycles. They were feed a commercial pellet diet and allowed access to water libitum. The experimental procedures were authorized by the institutional animal ethical committee.

The male albino rats were divided into three following category and each cluster contains six rats.

Group 1- Control rats treated with normal saline.

Group 2- SNPs $40 \mathrm{mg} / \mathrm{kg}$ body weight treated.

Group 3- SNPs $80 \mathrm{mg} / \mathrm{kg}$ body weight treated.

NOTE: The doses were given by intra peritoneal (IP) or by injection method alternatively.

\section{EXPERIMENTAL PROCEDURE}

After 14 days rats were allowed moving for behavioral test and sensory responses.

\section{Behavioral studies}

The Motor activity, behavioral changes, muscle coordination, sensory and motor reflex responses were assessed in SNPs administered rats and control rats. 
1. Spontaneous motor activity [SMA]: SMA was measured by scored on a scale of $0-9$ within which SMA up to the mark cluster was appointed score 4.

2. Righting reflex: Rats were placed on their back to check if the animal may quickly right itself and assume a common posture. Neurological deficits were indicating by associate degree inability to regain common body posture among 5 seconds.

3. Catalepsy: A condition within which body or limbs stay passively in any position in which they'll be placed and then this take a look at is employed by placing forepaws on a metallic rod placed at height of $6 \mathrm{~cm}$ and forepaws weren't observed withdrawn among $10 \mathrm{sec}$. catalepsy were thought of positive and if forepaws weren't withdrawn among $10 \mathrm{sec}$ than it thought of negative.

4. Muscle coordination test (Rota-rod): The amount of remain rotating-rod (speed: 5 rotations/min; Total period of take a look at a pair of min) for each and every rat (treated control and treated) were conformation by Rotamex (Techno Electronics, India). The rat was remaining on rotating-rod for 2 min and only trained rats were selected for the study. The motor was measured exploitation Rota-Rod a minimum of $5 \mathrm{~s}$ and it was absolutely revolved at speed of $10 \mathrm{rpm}$ for 2 consecutive days on third day the time period of each and every rotation speed was additionally recorded.

\section{Biochemical Estimations \\ Tissue Homogenate Preparation}

After 14 days the experimental rats were given anesthetic dose (sodium penta-barbital) and then dissected out from cranium and brain was removed. $10 \%$ $(w / v)$ homogenate of the brain was ready to prepare and exploitation York's homogenizer fitted with Teflon plunger in $0.1 \mathrm{M}$ phosphate buffer $(\mathrm{pH}$ 7.1). The complete homogenate material was $1^{\text {st }}$ centrifuged at $2500 \mathrm{x} g$ for 10 minutes in a refrigerated centrifuge. The pellet consisting of nuclear fraction and cell rubble was discarded. The supernatant was more centrifuged at $11,000 \mathrm{x} \mathrm{g}$ for 15 minutes and mitochondrial fraction was separated. The clear supernatant was more centrifuged at 105,000 x g for 90 minutes and therefore the resultant supernatant was used for determinative enzyme activities.
The protein estimation was done by the method of (Lowery et al 1995) using standard solution of bovine serum albumin (BSA). Lipid peroxide level (LPO) was done by using spectrophotometer at $532 \mathrm{~nm}$ according the method of (Okhawa et al 1979) and expressed as $n$ mole MDA/g tissue. The superoxide dismutase (SOD: EC 1.15.1.1.) was done NADPHphenanzomethosulphate, nitrobluetertrazolin formazon inhibition reaction by using spectrophotometer at 560 $\mathrm{nm}$ by the method of (Mc Cord and Fridovich 1969). The activity of catalase (CAT: EC 1.111.6) was done by the method of (Aebi et al 1974) using hydrogen peroxide as substrate, the decomposition of $\mathrm{H}_{2} \mathrm{O}_{2}$ was followed at 240nm.Glutathione (GSH) were done (Ellman et al 1959 ) in de-proteinezed supernatant of homogenate with employing 5-5' dithiobes (2-nitrobenzioc acid) and recorded absorption at $412 \mathrm{~nm}$. The GSH content was expressed as $\mu$ mole /g tissue.

\section{RESULT AND DISCUSSION}

The several studies indicated that Silica nanoparticles changes the memory and learning ability. During this modern study, firstly, we assessed neuromuscular dysfunctioning and also the gross behavioral activity response i.e., SMA, Gait, Catalepsy and Rota-rod are shown in the Table-1. The muscles that square measure vital for maintaining the stable posture that involves trunk muscles and also known as postural muscles within the extremities. It's recommended which SiNPs changes the posture position in rats and it's going to ensure to SiNPs induced alteration in central and peripheral nervous system. Catalepsy is that the position that the rats are unable to measure correct outwardly obligatory postures. There's the specialty of the pharmacological proof for stimulation of dopaminergic neurons by noradrenergic neurons within the brain. During this modern study, it's been reported that the Catalepsy could be a symptom of bound nervous disorders or conditions, for example, Parkinson's disease, epilepsy and Protein kinase- $\mathrm{C}$ has been recommended as an intermediators of cataleptic behavior, and it's additionally been recommended that SiNPs changes the protein kinase activity in each adult and old recent rat brain. As evident by our study, we recommend that SiNPs induces Gait, spontaneous activity and cataleptic behavior and increased muscle incoordination recommends that reduced the rate of ionic exchange from cells.

Table-1: Gross Behavioral Responses after 14 days of SiNPs treated group

\begin{tabular}{|l|l|l|l|l|l|}
\hline & Control & $\mathbf{4 0 m g / k g}$ & $\mathbf{8 0 m g} / \mathbf{k g}$ & $\begin{array}{l}\boldsymbol{\chi 2} \text { - test, } \\
(\mathbf{D F}=\mathbf{1 5})\end{array}$ & $\mathbf{p}$-value \\
\hline SMA & 100 & 78 & 72 & $\mathrm{x} 2=9.4$ & $\mathrm{p}=0.0801$ \\
\hline Righting reflex & $\mathrm{ND}$ & 77 & 66 & $\mathrm{x} 2=20$ & $\mathbf{p = 0 . 0 0 1 2}$ \\
\hline Catalepsy & ND & 55.3 & 44 & $\mathrm{x} 2=14.3$ & $\mathrm{p}=0.0136$ \\
\hline Rota rod & 100 & 28 & 22 & $\mathrm{x} 2=7.7$ & $\mathbf{p}=\mathbf{1 7 3 6}$ \\
\hline
\end{tabular}


Table shows Gross behavioral responses after 14 days of treatment in each groups $(n=6)$. Data were analyzed by $\chi^{2}$ test and express as $\%$ of rats represented characters

The SMA was found to be considerably $(p<0.05)$ reduced in SiNPs 80 when it compared with the controls. Catalepsy was present in both treated groups. The SiNPs treated rats were unable to stay in metallic rod of more than 10 seconds. The significant reduction of time was observed in both SiNPs 80 and SNPs 40 treated rats. Gait was also found to be reduced time to maintain posture in the edge of the cage. Both treated groups were showed significant $(\mathrm{p}<0.05)$ change when it compared with the control. The muscles coordination was performed using Rota-rod test. The time of staying at rod was found to be considerably reduced in each SiNPs 80 and SiNPs 40 groups once it analyze with their respective controls. The maximum behavioral changes found in $80 \mathrm{mg} / \mathrm{kg}$ body weight of SiNPs treated rats.

The behavioral observation was to calculate the potential toxicity and the general mechanism was consumed in SiNPs induced cell toxicity. During this modern study was the cell toxicity of two different doses (40 and $80 \mathrm{mg}$ ), it absolutely found that the exposure of Silica nanoparticles at dose levels characterized in different neurochemical changes. We recognized that SiNPs induced neurochemical alteration in rats and it relates within the dose dependent association of and neurobehavioral findings. The body weight is gain by the effects of Silica, when food intake and feed potency was increasingly increased throughout the experimental period of each the category. The ultimate body weight of intoxicated rats (Fig-1) with the Silica was considerably not up to the healthy common category of rats. The results indicated the SiNPs cause significantly decreases body weight of treated rats. That the dangerous effects of SiNPs on the weight boosting were highly dignified paralleled with increase of SiNPs dose and it absolutely suggestive that the loss of body mass showed that SiNPs induces several toxicity and it might be due to the loss of lipid, protein and other biomolecules. The increased muscle incoordination recommends that reduced the rate of ionic exchange from cells. Our results additionally incontestable per-oxidative harmful for brain, as measured by extend of the amount of macromolecules lipid peroxidation products (MDA). This might ensure to extend the speed of ROS manufacturing. Also, the membrane contains an oversized quantity of lipids that square measure made in polyunsaturated fatty acids; they'll additionally promptly reacts with free radicals and undergo peroxidation. Although the SiNPs in biological tissues haven't got any direct pro-oxidant properties however they'll raise the elements to raise the ROS development and improve the per-oxidative harm to lipids and proteins.

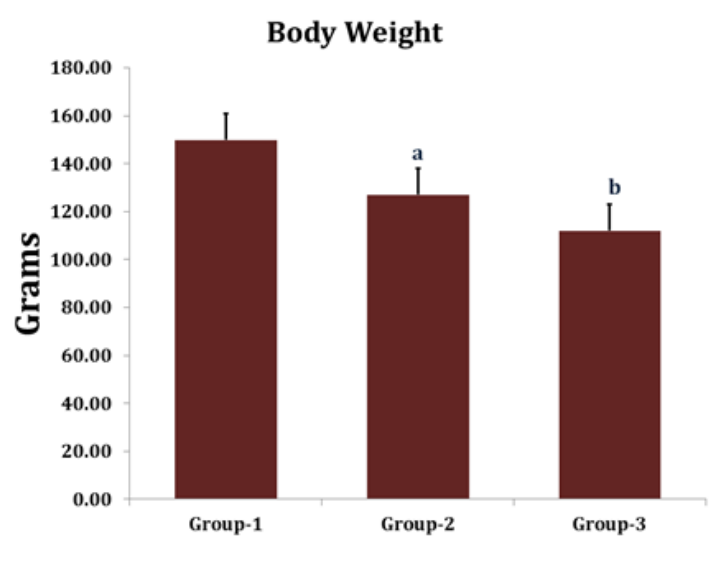

Fig-1: The body weight of control and experimental rats are expressed as mean \pm SEM for six animals $(N=6)$ in each group. Body weight in grams of SiNPs $40 \mathrm{mg}$ and $80 \mathrm{mg}$ treated and control rats. Statistical significance was determined by One way ANOVA followed by Student Newman-Kules test. Probability, p-value less than 0.05 were considered statistically significant.

Cellular endogenous resist the system possess several inhibitor enzymes, among them the SOD plays a significant role because it prevents the ROS mediated tissue or cell harm and catalase are the vital antioxidants, and also within the cells that defend it from $\mathrm{H}_{2} \mathrm{O}_{2}$ mediated cellular harm and thus reduced the amount of those biomolecules, also it causes to the raised severity of doses which dependent upon the SiNPs toxicosis. The many mechanisms are planned to clarify the adverse health effects of the particulate toxicity and also the ROS manufacture and also the originations of oxidative stress have received the most attention. For example the ROS have, super oxides, hydrogen peroxide, hydroxyl and other oxygen radicals, which capable of immediately oxidizing the DNA, proteins, and lipids (Yoshida et al., 2004). They have several confirmations which display the nanoparticles increased the ROS manufacturing and it causes the apoptosis in various groups of cultured cells (Park et al., 2008). Further, it's been well recorded that antioxidant depletion and also the ROS manufacture that causes the mitochondrial dysfunction and converting the expression of distinct genes and also the pathways involving inflammatory responses and apoptosis is get together with the MAPK/ERK kinase, NFjB, MIP-2, caspase-3, Bcl-2 (Kharasch et al., 2006). Consequently, the apoptosis started by silicon dioxide is also the results of the ROS manufacturing is raised and antioxidant depletion, resulting in mitochondrial dysfunction, DNA damage, raised gene expression of death receptors as well as their corresponding ligands. During, the modern studies are expected to investigate the expression of genes in these signaling pathways in response to exposure of $\mathrm{SiO}_{2}$ nanoparticles. The decrement in SOD or CAT levels indicate increased oxidative burden, whereas an imbalance between SOD and CAT activities could lead to an excessive generation of free radicals. The anti-oxidative defense system like SOD and CAT 
showed lower activities in brain during toxicity. Reduced glutathione $(\mathrm{GSH})$ is central to the cellular antioxidant defences and acts as an important cofactor for antioxidant enzymes (Hayes et al., 2005). Under oxidative stress, glutathione is consumed by the glutathione-related enzymes to detoxify peroxides produced because of increased LPO, and elevation in LPO is a consequence of depleted GSH stores, which are otherwise capable of moderating the amount of LPO. Similarly, Silica oral induced disturbances in the antioxidant barrier (involving glutathione peroxidase and superoxide dismutase) have been shown increment of one enzyme was accompanied with the depletion of the other enzyme. The brain have various antioxidants like SOD, Catalase, GSH, GR etc and SOD is the major enzymes by which it protects tissue damage by ROS as well as glutathione help in detoxification of ROS in body. The level of GSH in different region of brain was decreased (Liu et al 2003).

In summary, our preliminary information has been recommended that exposure of Silica nanoparticles results in the cellular morphological modifications, mitochondrial dysfunction, and oxidative stress as indicated by elevation of eliminate thing ROS and TBARS, at least of depletion of antioxidant that triggers cell cycle arrest and apoptosis in a dose dependent manner.

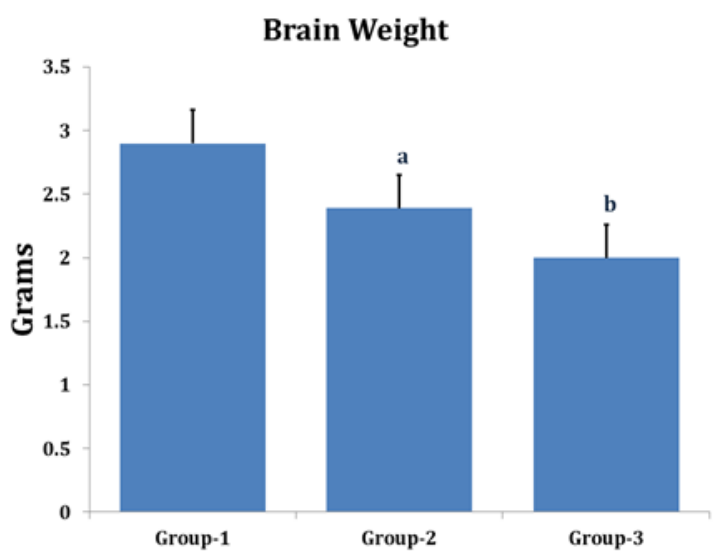

Fig-2: The weights of the brain are presented as mean \pm SEM in controls and experimental groups following 14 days of SiNPs administration. Data were analyzed by One way ANOVA followed by Student Newman-Kules test. The significant comparison represent between control and SiNPs $40 \mathrm{mg}$ treated rats (a) and control treated with SiNPs $80 \mathrm{mg}$ (b).The $P$ value $<0.05$ was considered significant.
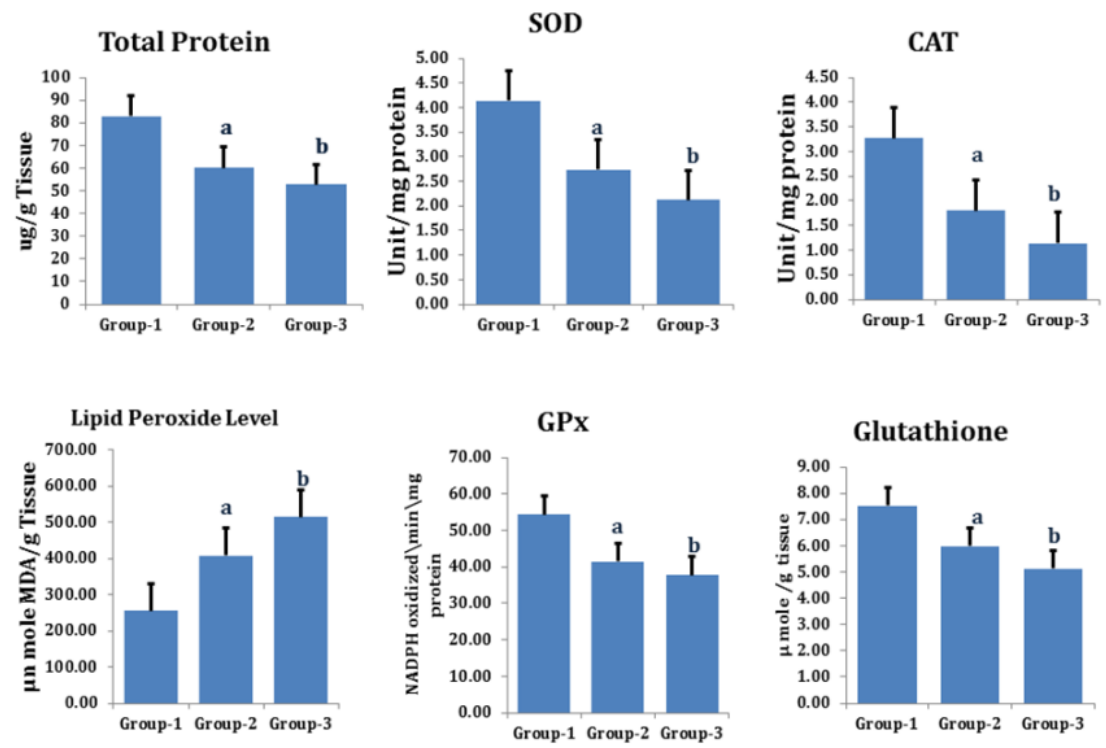

Fig-3: Protein estimation, Lipid peroxide level, Superoxide dismutase, Catalase, GPx and GSH levels in brain of control and SiNPs treated group. The results are expressed as Mean \pm SEM in six rat of each group $(\mathrm{N}=6)$. Superscripts relate significant $(\mathbf{p}<0.05)$ comparison with Control and treated group.

\section{CONCLUSION}

The present study concludes that silica induced neurotoxicity by free radical mechanism. In summary, our primary information's has recommended that exposure of Silica nanoparticles results to cellular morphological modifications, mitochondrial dysfunction, and oxidative stress as showed by rise of intracellular ROS and TBARS, and in addition exhaustion of cancer prevention agent (SOD and CAT), which may triggers neuronal damage further it promotes to cognitive decline. Further, this study is required to reveal the mechanism of SNPs in different models.

\section{ACKNOWLEDGEMENT}

The authors would like to acknowledge Nims University Rajasthan, Jaipur for research funding. 


\section{REFERENCES}

1. Abbott, N. J., Rönnbäck, L., \& Hansson, E. (2006). Astrocyte-endothelial interactions at the blood-brain barrier. Nature reviews neuroscience, 7(1), 41-53.

2. Aebi, H., Wyss, S. R., Scherz, B., \& SKVARIL, F. (1974). Heterogeneity of erythrocyte catalase II: isolation and characterization of normal and variant erythrocyte catalase and their subunits. European Journal of Biochemistry, 48(1), 137-145.

3. Cole, J. C., \& Sumnall, H. R. (2003). The pre-clinical behavioural pharmacology of 3 , 4-methylenedioxymethamphetamine (MDMA). Neuroscience \& Biobehavioral Reviews, 27(3), 199-217.

4. De Jong, W. H., \& Borm, P. J. (2008). Drug delivery and nanoparticles: applications and hazards. International journal of nanomedicine, 3(2), 133.

5. Ellman, G. L. (1959). Tissue sulfhydryl groups. Archives of biochemistry and biophysics, 82(1), 70-77.

6. Gustavsson, A., Svensson, M., Jacobi, F., Allgulander, C., Alonso, J., Beghi, E., ... \& CDBE2010 Study Group. (2011). Cost of disorders of the brain in Europe 2010. European neuropsychopharmacology, 21(10), 718-779.

7. Hayes, J. D., Flanagan, J. U., \& Jowsey, I. R. (2005). Glutathione transferases. Annu. Rev. Pharmacol. Toxicol., 45, 51-88.

8. Kharasch, E. D., Schroeder, J. L., Bammler, T., Beyer, R., \& Srinouanprachanh, S. (2006). Gene expression profiling of nephrotoxicity from the sevoflurane degradation product fluoromethyl-2, 2-difluoro-1-(trifluoromethyl) vinyl ether ("compound A”) in rats. Toxicological Sciences, 90(2), 419-431.

9. Kim, I. Y., Joachim, E., Choi, H., \& Kim, K. (2015). Toxicity of silica nanoparticles depends on size, dose, and cell type. Nanomedicine: Nanotechnology, Biology and Medicine, 11(6), 1407-1416.

10. Kontoleontos, F., Tsakiridis, P. E., Marinos, A., Kaloidas, V., \& Katsioti, M. (2012). Influence of colloidal nanosilica on ultrafine cement hydration: Physicochemical and microstructural characterization. Construction and building materials, 35, 347-360.

11. Liu, R., Liu, I. Y., Bi, X., Thompson, R. F., Doctrow, S. R., Malfroy, B., \& Baudry, M. (2003). Reversal of age-related learning deficits and brain oxidative stress in mice with superoxide dismutase/catalase mimetics. Proceedings of the National Academy of Sciences, 100(14), 8526-8531.

12. Lowry, O. H., Rosebrough, N. J., Farr, A. L., \& Randall, R. J. (1951). Protein measurement with the Folin phenol reagent. Journal of biological chemistry, 193, 265-275.

13. McCord, J. M., \& Fridovich, I. (1969). Superoxide dismutase: an enzymic function for erythrocuprein (hemocuprein). Journal of Biological chemistry, 244(22), 6049-6055.

14. Nakamura, T., Sugihara, F., Matsushita, H., Yoshioka, Y., Mizukami, S., \& Kikuchi, K. (2015). Mesoporous silica nanoparticles for $19 \mathrm{~F}$ magnetic resonance imaging, fluorescence imaging, and drug delivery. Chemical science, 6(3), 1986-1990.

15. Nel, A., Xia, T., Mädler, L., \& Li, N. (2006). Toxic potential of materials at the nanolevel. science, 311(5761), 622-627.

16. Niu, Y. M., Zhu, X. L., Chang, B., Tong, Z. H., Cao, W., Qiao, P. H., ... \& Song, Y. G. (2016). Nanosilica and polyacrylate/nanosilica: a comparative study of acute toxicity. BioMed research international, 2016.

17. Ohkawa, H., Ohishi, N., \& Yagi, K. (1979). Assay for lipid peroxides in animal tissues by thiobarbituric acid reaction. Analytical biochemistry, 95(2), 351-358.

18. Park, E. J., \& Park, K. (2009). Oxidative stress and pro-inflammatory responses induced by silica nanoparticles in vivo and in vitro. Toxicology letters, 184(1), 18-25.

19. Passeleu-Le Bourdonnec, C., Carrupt, P. A., Scherrmann, J. M., \& Martel, S. (2013). Methodologies to assess drug permeation through the blood-brain barrier for pharmaceutical research. Pharmaceutical research, 30(11), 2729-2756.

20. Pisani, C., Gaillard, J. C., Nouvel, V., Odorico, M., Armengaud, J., \& Prat, O. (2015). High-throughput, quantitative assessment of the effects of low-dose silica nanoparticles on lung cells: grasping complex toxicity with a great depth of field. BMC genomics, 16(1), 1-14.

21. Sanchez, F., \& Sobolev, K. (2010). Nanotechnology in concrete-a review. Construction and building materials, 24(11), 2060-2071.

22. Ungvari, Z., Bailey-Downs, L., Gautam, T., Jimenez, R., Losonczy, G., Zhang, C., ... \& Csiszar, A. (2011). Adaptive induction of NF-E2-related factor-2-driven antioxidant genes in endothelial cells in response to hyperglycemia. American Journal of Physiology-Heart and Circulatory Physiology, 300(4), H1133-H1140.

23. Xiao, X., Liu, Y., Guo, M., Fei, W., Zheng, H., Zhang, R., ... \& Li, F. (2016). pH-triggered sustained release of arsenic trioxide by polyacrylic acid capped mesoporous silica nanoparticles for solid tumor treatment in vitro and in vivo. Journal of biomaterials applications, 31(1), 23-35.

24. Yang, Y., \& Li, J. (2014). Lipid, protein and poly (NIPAM) coated mesoporous silica nanoparticles for biomedical applications. Advances in colloid and interface science, 207, 155-163.

25. Yoshida, Y., Itoh, N., Saito, Y., Hayakawa, M., \& Niki, E. (2004). Application of water-soluble radical initiator, 2, 2'-azobis-[2-(2-imidazolin-2-yl) propane] dihydrochloride, to a study of oxidative stress. Free radical research, 38(4), 375-384.

26. Zhang Q, Kusaka Y, Sato K, Nakakuki K, Kohyama N, Donaldson K. (2012). Differences in the extent of inflammation caused by intratracheal exposure to three ultrafine metals: Role of free radicals. J Toxicol Environ Health A., 53; 423-438.

27. Zielinski, J., Möller, A. M., Frenz, M., \& Mevissen, M. (2016). Evaluation of endocytosis of silica particles used in biodegradable implants in the brain. Nanomedicine: Nanotechnology, Biology and Medicine, 12(6), 1603-1613. 